\title{
Micobactérias não-tuberculosas: diversidade das espécies no estado de São Paulo
}

\author{
Nontuberculous mycobacteria: species diversity in São Paulo state, Brazil
}

Suely Yoko Mizuka Ueki'; Maria Conceição Martins²; Maria Alice da Silva Telles³; Melissa Curcio Virgilio4; Carmen Maria Saraiva Giampaglia ${ }^{5}$ Erica Chimara ${ }^{6}$; Lucilaine Ferrazoli

\begin{abstract}
unitermos resumo
Micobactérias não-tuberculosas

(MNT)

Diagnóstico bacteriológico

Complexo Mycobacterium avium

Mycobacterium kansasii

O gênero Mycobacterium é constituído por espécies do complexo $M$. tuberculosis e outras denominadas micobactérias não-tuberculosas (MNT). Até o momento, mais de cem MNT foram descritas. Os objetivos deste estudo foram avaliar a diversidade das espécies de MNT identificadas no estado de São Paulo, no período de 1991 a 1997, que antecedeu a expansão da terapia anti-retroviral, e determinar a freqüência dos casos que atenderam alguns critérios bacteriológicos para o diagnóstico das infecções causadas pelas MNT. Material e métodos: Foram analisadas 1.892 cepas isoladas de sítios estéreis e não-estéreis de 1.248 pacientes atendidos no estado de São Paulo. Resultados: Do total de pacientes, 1.199 (96,1\%) tiveram suas cepas identificadas e 3,9\% apresentaram resultados não-conclusivos. As dez espécies encontradas foram o complexo M. avium (MAC), M. kansasii, M. chelonae, M. fortuitum, M. szulgai, M. xenopi, M. marinum, M. gordonae, M. terrae e M. nonchromogenicum. Quarenta e sete $(7,8 \%)$ casos pulmonares tiveram diagnóstico confirmado pelo isolamento da mesma espécie em três ou mais amostras e 67 (34\%) pacientes tiveram o diagnóstico bacteriológico confirmado por isolamento em sítios estéreis. Conclusões: As espécies de MNT mais freqüentemente isoladas no estado de São Paulo foram MAC e M. kansasii. Uma publicação nacional com recomendações para diagnóstico e tratamento dessas infecções seria fundamental para a conduta correta no diagnóstico e no tratamento de micobacterioses.
\end{abstract}

Introduction: Mycobacterium genus includes species of M. tuberculosis complex and others called non-tuberculous mycobacteria (NTM). So far, more than 100 species of NTM have been described. The objectives of this study were to estimate the NTM species diversity among strains isolated in São Paulo State from 1991 to 1997, before the expansion of antiretroviral therapy, and to evaluate the rate of cases that fall under diagnostic criteria for bacteriological diagnosis of NTM infections. Material and methods: 1,892 strains were isolated from sterile and non-sterile sites of 1,248 patients. Results: 1,199 (96\%) were identified as M. avium complex (MAC), M. kansasii, M. chelonae, M. fortuitum, M. szulgai, M. xenopi, M. marinum, M. gordonae, M. terrae or M. nonchromogenicum whereas 49 (3.9\%) couldn't be identified up to species. Forty-seven (7,8\%) cases from pulmonary sites were confirmed based on strains isolated from three or more samples from the same patient and 67 (34\%) patients had NTM isolated from sterile sites, which confirmed the bacteriological diagnosis. Conclusions: MAC and M. kansasii were the most frequently especies isolated in São Paulo state. National recomendations for the diagnosis and treatment of NTM infections would be useful for correct management of NTM infections.

\section{key words} Nontuberculous mycobacteria (NTM)

Bacteriological diagnostic

Mycobacterium avium

complex

Mycobacterium kansasii

1. Especialista em Saúde Pública; pesquisadora científica do Instituto Adolfo Lutz.

2. Mestra em Saúde Pública; pesquisadora científica do Instituto Adolfo Lutz.

3. Especialista em Saúde Pública; pesquisadora científica encarregada do Setor de Micobactérias do Instituto Adolfo Lutz.

4. Cerente de produtos do Diagnostic Systems da Becton-Dickinson.

5. Mestra em Microbiologia e Imunologia; pesquisadora científica do Instituto Adolfo Lutz.

6. Mestra em Microbiologia; pesquisadora científica do Instituto Adolfo Lutz.

7. Doutora em Microbiologia; pesquisadora científica do Instituto Adolfo Lutz.

Trabalho realizado no Setor de Micobactérias do Instituto Adolfo Lutz, laboratório central. 


\section{Introdução}

O gênero Mycobacterium é constituído por espécies do complexo M. tuberculosis (M. tuberculosis, M. bovis, M. bovisBCG, M. africanum, M. microti) e outras que atualmente são denominadas micobactérias não-tuberculosas (MNT) $)^{(10,13,}$ 27). Até o momento mais de cem MNT foram descritas ${ }^{(13)}$. Essas espécies, geralmente oportunistas, são classificadas, conforme sua capacidade de causar doença no homem, como potencialmente patogênicas e não-patogênicas ${ }^{(4,}$ $10,11)$.

As MNT potencialmente patogênicas podem causar diversas formas de doença, inclusive a disseminada, acometendo pulmões, gânglios, pele, articulações. Essas infecções, se não tratadas oportunamente, podem ser fatais ou causar seqüelas graves $(6,7,20,25)$. Entre as espécies potencialmente patogênicas, as pertencentes ao complexo M. avium (MAC) e M. kansasii são as mais freqüentemente isoladas $(5,6,14,16,22)$.

Vários estudos foram publicados estabelecendo critérios para o diagnóstico das infecções causadas pelas MNT, pois essas espécies podem colonizar transitoriamente 0 homem ${ }^{(4)}$. Embora exista certa variação entre esses critérios, todos enfatizam a importância do isolamento repetido do mesmo agente a partir de espécimes biológicos nãoestéreis ou obtenção de um cultivo puro de biópsias e/ou outros fluidos de sítios supostamente estéreis. Os critérios aplicam-se a pacientes com sinais clínicos, radiológicos e/ou histopatológicos compatíveis com um quadro de infecção ${ }^{(3,}$ $4,8,11,16,18,20$ )

Até 1980 a maioria dos casos descritos sobre infecções causadas por MNT referiam-se a doenças pulmonares causadas por $M$. kansasii e MAC, doenças cutâneas por $M$. marinum e linfadenopatias cervicais por M. scrofulaceum ${ }^{(1,}$ 14). Posteriormente, as pesquisas feitas na década de 1990 demonstraram aumento nas taxas de isolamento das MNT. Estudo realizado pelo Center for Disease Control (CDC) nos anos de 1991 e 1992, incluindo 33 laboratórios, mostrou elevação nas taxas de isolamento de MAC quando em comparação com as de M. tuberculosis, que representavam apenas $26 \%{ }^{(4)}$. A pandemia da síndrome de imunodeficiência adquirida (AIDS) foi a que mais contribuiu para este aumento, principalmente da doença disseminada por



No Brasil, na década de 1990, foram realizados estudos sobre a ocorrência das MNT, alguns deles em pacientes portadores do vírus da imunodeficiência humana (HIV). Em São Paulo, Barreto et al.(6) demonstraram que MNT foram isoladas de medula óssea em $20 \%$ dos pacientes estudados; Ferreira et al., no período entre 1996 e 1997, no Rio de Janeiro, relataram 15\% de casos de MNT e, entre estes, 57,8\% de MAC; Fandinho et al. ${ }^{(15)}$, no Rio de Janeiro, detectaram $37 \%$ de MAC em hemocultura de pacientes HIV-positivos ${ }^{(6,15,17)}$.

O Setor de Micobactérias do Instituto Adolfo Lutz, como referência estadual para micobactérias, recebe cepas de todo o estado de São Paulo para identificação e teste de sensibilidade às drogas. Portanto, o número e as espécies identificadas refletem, ainda que não em sua totalidade, a diversidade dessas espécies isoladas de espécimes clínicos no estado.

Os objetivos deste estudo foram determinar a diversidade das espécies identificadas no estado de São Paulo durante o período de 1991 a 1997, que antecedeu a expansão da highly active antiretroviral therapy (HAART), e a freqüência dos casos que atenderam a alguns critérios bacteriológicos para o diagnóstico das infecções causadas por MNT.

\section{Material e método}

\section{Cepas estudadas}

No período de 1991 a 1997 foram analisadas 2.053 cepas de micobactérias isoladas de 1.267 pacientes atendidos nos serviços de saúde pública das diversas regiões do estado de São Paulo. Elas foram obtidas de diversos espécimes biológicos coletados de sítios estéreis e não-estéreis, sendo que em alguns casos os isolamentos tiveram origem em dois ou mais sítios.

\section{Identificação fenotípica}

As cepas foram submetidas a triagem por análises macroscópica e microscópica da cultura que permitissem separar as espécies sugestivas de pertencerem ao complexo M. tuberculosis das MNT. A morfologia e a pigmentação das colônias foram as características macroscópicas avaliadas. Com a análise microscópica do esfregaço das colônias corado pelo método de Ziehl-Neelsen, foram avaliadas a morfologia do bacilo álcool-ácido-resistente (BAAR), a formação de corda e a presença de contaminação por outras bactérias e fungos ${ }^{(10)}$. As cepas com colônias pigmentadas ou acromógenas lisas que não apresentaram formação de corda no exame microscópico tiveram classificação presuntiva de MNT e foram submetidas aos testes fenotípicos para identificação da espécie, como análise do tempo e da temperatura de crescimento e testes enzimáticos e de 
inibição de crescimento na presença de drogas ${ }^{(10,21,27)}$. As cepas que apresentaram resultados não-conclusivos nos testes foram classificadas, de acordo com o tempo de crescimento e a pigmentação, em micobactéria de crescimento lento acromógena (CLA), de crescimento lento escotocromógena (CLE), de crescimento lento fotocromógena (CLF), de crescimento rápido acromógena (CRA), de crescimento rápido escotocromógena (CRE) e de crescimento rápido fotocromógena (CRF).

\section{Análise dos dados}

A análise foi feita a partir de um banco de dados construído com o programa de computador EPIINFO 6.04C (CDC, Atlanta), no qual foram digitadas as informações contidas nos registros laboratoriais (data de isolamento, espécime clínico, sexo e HIV) e os resultados da identificação da espécie. A origem dos espécimes clínicos foi classificada como pulmonar, extrapulmonar e disseminada. Escarro, lavado brônquico e lavado gástrico foram classificados como de origem pulmonar. Os líquidos cefalorraquidiano e pleural, a urina, as biópsias (gânglio, pele, fígado, estômago) e as fezes foram classificadas como de origem extrapulmonar. Os isolamentos obtidos a partir de sangue e/ou medula óssea, ou os de dois sítios estéreis, foram classificados como de origem disseminada.

\section{Resultados}

No período de sete anos de estudo, foram analisadas 2.053 cepas de MNT isoladas de 1.267 pacientes. Dezenove pacientes e 161 cepas foram excluídos da análise, pois mais de uma espécie de micobactéria foi isolada em diferentes espécimes clínicos. Dessa forma, foram analisadas 1.892 cepas de MNT isoladas de 1.248 pacientes. Desses, 950 (76,1\%) eram do sexo masculino e 298 (23,9\%), do feminino. Cepas de $812(65,1 \%)$ pacientes foram recebidas de laboratórios da região metropolitana da Grande São Paulo, $428(34,3 \%)$ do interior do estado e oito $(0,6 \%)$ tinham procedência ignorada.

As cepas isoladas de $1.199(96,1 \%)$ pacientes foram identificadas até a espécie, enquanto aquelas originadas dos 49 (3,9\%) restantes apresentaram resultados não-conclusivos. Quarenta e oito cepas foram classificadas em grupos de acordo com o tempo de crescimento e a pigmentação, sendo que um paciente apresentou cultura mista, o que não permitiu sua classificação.

Entre as cepas identificadas foram encontradas dez espécies, entre elas sete potencialmente patogênicas (MAC,
M. kansasii, M. chelonae, M. fortuitum, M. szulgai, M. xenopi, M. marinum) e três não-patogênicas (M. gordonae, M. terrae, M. nonchromogenicum). Entre as espécies identificadas, três foram mais freqüentes: MAC $(64,9 \%), M$. kansasii $(12,2 \%)$ e M. gordonae (10,2\%). A distribuição anual dessas espécies mostrou que, de 1993 a 1996, houve um aumento nas suas taxas de isolamento, com ligeiro declínio em 1997 (Tabela 1).

Dos 1.248 pacientes, 821 (65,8\%) tinham informação quanto ao HIV. Do total de pacientes, $744(59,6 \%)$ eram positivos, 77 (6,2\%) negativos e 427 (34,2\%) não apresentaram essa informação. As freqüências de MAC $(73,7 \%)$ e M. kansasii (49\%) foram maiores entre os pacientes HIVpositivos quando em comparação com as encontradas nos negativos. Os quatro casos de M. xenopi ocorreram somente entre pacientes HIV positivos (Tabela 2).

A distribuição das espécies identificadas segundo os sítios de isolamento demonstrou que 600 (48\%) pacientes tiveram isolamento de MNT de origem pulmonar; 366 (29,3\%), disseminada; e 197 (15,8\%), extrapulmonar. O isolamento do MAC $(44,2 \%)$ foi mais freqüente na forma disseminada, e do $M$. kansasii (83\%), na pulmonar. Seis pacientes tiveram $M$. marinum isolados de sítios extrapulmonares, cinco de biópsias de pele e um de gânglio. Das 48 cepas que apresentaram resultados não-conclusivos na identificação, $31(64,6 \%)$ foram isoladas de sítios pulmonares (Tabela 3).

A Tabela 4 mostra o número de cepas isoladas por paciente e os respectivos sítios de origem. Entre os isolamentos de sítios não-estéreis, 479 (79,8\%) de origem pulmonar e $119(93,7 \%)$ de extrapulmonar tiveram apenas um isolamento analisado.

\section{Discussão}

As doenças causadas pelas MNT aumentaram em todo o mundo no período pós-AIDS. Os estudos realizados nos Estados Unidos e na Europa relataram 25\% a 60\% de infecções por MNT entre pacientes portadores de $\operatorname{HIV}^{(1,4,9,14}$, ${ }^{19,20)}$. No Brasil, as doenças causadas pelas MNT não são de notificação obrigatória, portanto não há registros oficiais para que se possa estimar sua incidência. Os estudos feitos entre pacientes portadores de HIV demonstraram variação entre $15 \%$ e $37 \%$ nas taxas de isolamento de $\mathrm{MNT}^{(6,15,17)}$. No presente estudo, nos anos de 1993 a 1996, foi observado um aumento na identificação das duas espécies potencialmente patogênicas MAC e M. kansasii. Esse fato pode ter sido influenciado pela realização de um número maior 
Tabela 1 Distribuição anual das espécies de MNT identificadas no estado de Sáo Paulo de 1991 a 1997

\begin{tabular}{lcccccccc}
\hline Espécie & $n(\%)$ & & & & Ano & & \\
MAC & Total & 91 & 92 & 93 & 94 & 95 & 96 & 97 \\
M. kansasii & $810(64,9)$ & 118 & 101 & 73 & 104 & 141 & 163 & 110 \\
M. gordonae & $153(12,2)$ & 18 & 10 & 8 & 12 & 24 & 43 & 38 \\
M. fortuitum & $128(10,2)$ & 8 & 12 & 3 & 13 & 31 & 37 & 24 \\
M. terrae & $49(3,9)$ & 10 & 4 & 5 & 5 & 11 & 7 & 7 \\
M. chelonae & $24(1,9)$ & 1 & 5 & 3 & 8 & 4 & 1 & 2 \\
M. marinum & $21(1,7)$ & 2 & 2 & 4 & 5 & 1 & 4 & 3 \\
M. xenopi & $6(0,5)$ & - & - & 1 & - & 2 & 2 & 1 \\
M. nonchromogenicum & $4(0,4)$ & 1 & - & - & 2 & 1 & - & - \\
M. szulgai & $3(0,2)$ & 3 & - & - & - & - & - & - \\
CRA & $1(0,1)$ & - & - & - & - & - & - & 1 \\
CRE & $34(2,7)$ & 1 & - & - & 7 & 4 & 9 & 13 \\
CRF & $8(0,6)$ & - & 1 & - & - & 3 & 3 & 1 \\
CLF & $2(0,2)$ & - & - & - & - & 1 & 1 & - \\
CLE & $2(0,2)$ & - & - & - & - & 2 & - & - \\
CLA & $1(0,1)$ & - & - & - & - & 1 & - & - \\
Cultura mista & $1(0,1)$ & - & - & - & - & - & - & 1 \\
Total & $1(0,1)$ & - & - & 1 & - & - & - & - \\
\hline
\end{tabular}

$M A C=$ complexo M. avium; $C L A=$ micobactéria de crescimento lento acromógena; $C R A=$ micobactéria de crescimento rápido acromógena; $C L F=$ micobactéria de crescimento lento fotocromógena; $C L E=$ micobactéria de crescimento lento escotocromógena; $C R E=$ micobactéria de crescimento rápido escotocromógena; $C R F=$ micobactéria de crescimento rápido fotocromógena; cultura mista = duas espécies de micobactérias isoladas de um único espécime biológico.

\section{Tabela 2 Distribuição dos 1.248 pacientes segundo espécies de MNT e HIV de 1991 a 1997}

\begin{tabular}{lccc}
\hline & & $n(\%)$ HIV & \\
Espécie & Positivo & Negativo & Ignorado \\
& $(n=744)$ & $(n=77)$ & $(n=427)$ \\
MAC & $597(73,7)$ & $22(2,7)$ & $191(23,6)$ \\
M. kansasii & $75(49)$ & $26(17)$ & $52(34)$ \\
M. gordonae & $27(21,1)$ & $14(10,9)$ & $87(68)$ \\
M. fortuitum & $14(28,6)$ & $3(6,1)$ & $32(65,3)$ \\
M. terrae & $4(16,7)$ & $2(8,3)$ & $18(75)$ \\
M. chelonae & $8(38)$ & $3(14,3)$ & $10(47,7)$ \\
M. marinum & $2(33,3)$ & $3(50)$ & $1(16,7)$ \\
M. xenopi & $4(100)$ & - & - \\
M. nonchromogenicum & - & $1(33,3)$ & $2(66,7)$ \\
M. szulgai & - & - & $1(100)$ \\
CRA & $9(26,5)$ & $2(5,9)$ & $23(67,6)$ \\
CRE & $1(12,5)$ & - & $7(87,5)$ \\
CRF & $1(50)$ & $1(50)$ & - \\
CLF & - & - & $2(100)$ \\
CLE & $1(100)$ & - & - \\
CLA & - & - & $1(100)$ \\
Cultura mista & $1(100)$ & - & - \\
\hline
\end{tabular}

$M A C=$ complexo M. avium; $C L A=$ micobactéria de crescimento lento acromógena; $C R A=$ micobactéria de crescimento rápido acromógena; $C L F=$ micobactéria de crescimento lento fotocromógena; $C L E$ = micobactéria de crescimento lento escotocromógena; $C R E$ = micobactéria de crescimento rápido escotocromógena; $C R F=$ micobactéria de crescimento rápido fotocromógena; cultura mista = duas espécies de micobactérias isoladas de um único espécime biológico. 
Distribuição das espécies de micobactérias isoladas segundo a origem do sítio de isolamento Tabela 3 de 1991 a 1997

\begin{tabular}{lccccc}
\hline Espécie & Pulmonar & Extrapulmonar & Origem & Disseminada & Outras* \\
$(n=600)$ & $(n=197)$ & $(n=366)$ & $(n=4)$ & $\begin{array}{c}\text { Ignorada } \\
(n=81)\end{array}$ \\
MAC & $308(38)$ & $92(11,4)$ & $358(44,2)$ & $4(0,5)$ & $48(5,9)$ \\
M. kansasii & $127(83)$ & $11(7,2)$ & $4(2,6)$ & - & $11(7,2)$ \\
M. gordonae & $69(53,9)$ & $52(40,6)$ & - & - & $7(5,5)$ \\
M. fortuitum & $30(61,2)$ & $16(32,6)$ & - & - & $3(6,2)$ \\
M. terrae & $19(79,2)$ & $3(12,5)$ & - & - & $2(8,3)$ \\
M. chelonae & $13(62)$ & $5(23,8)$ & $1(4,8)$ & - & $2(9,4)$ \\
M. marinum & - & $6(100)$ & - & - & - \\
M. xenopi & $1(25)$ & - & $2(50)$ & - & $1(25)$ \\
M. nonchromogenicum & - & $2(66,6)$ & - & - & $1(33,4)$ \\
M. szulgai & $1(100)$ & - & - & - & - \\
CRA & $22(64,7)$ & $7(20,6)$ & $1(2,9)$ & - & $1(11,8)$ \\
CRE & $6(75)$ & $1(12,5)$ & - & - & $1(50)$ \\
CRF & $1(50)$ & - & - & - & - \\
CLF & $1(50)$ & $1(50)$ & - & - & - \\
CLE & $1(100)$ & - & - & - & - \\
CLA & - & $1(100)$ & - & - & - \\
Cultura mista & $1(100)$ & - & - & - & - \\
\hline
\end{tabular}

$M A C=$ complexo M. avium; $C L A=$ micobactéria de crescimento lento acromógena; $C R A=$ micobactéria de crescimento rápido acromógena; $C L F=$ micobactéria de crescimento lento fotocromógena; $C L E$ = micobactéria de crescimento lento escotocromógena; $C R E$ = micobactéria de crescimento rápido escotocromógena; $C R F=$ micobactéria de crescimento rápido fotocromógena; cultura mista = duas espécies de micobactérias isoladas de um único espécime biológico. Pulmonar: escarro, lavado gástrico, lavado brônquico. Disseminada: sangue e/ou medula ou dois sítios estéreis. Extrapulmonar: biópsia de gânglio, fígado, estômago, pele, líquido cefalorraquidiano, líquido pleural, urina, fezes, outros.

*Isolamentos obtidos de dois sítios não-estéreis e de um sítio estéril e outro não-estéril.

de culturas para micobactérias pelos laboratórios públicos e pelo aumento do número de pacientes portadores de $\mathrm{HIV}^{(2,}$ 24). A queda nas taxas de isolamento no ano de 1997 pode ter ocorrido em razão da expansão da terapia HAART no estado de São Paulo, em novembro de $1996^{(12)}$.

O impacto do aumento de casos de HIV/AIDS no estado de São Paulo também foi observado neste estudo. Dos 821 pacientes que dispunham dessa informação, 744 (90,6\%) eram HIV-positivos. Das dez espécies identificadas, sete foram mais freqüentes entre os pacientes HIV-positivos. A predominância das duas espécies potencialmente patogênicas diferiu entre os pacientes HIV-positivos e negativos, e a taxa de isolamento de MAC $(73,7 \%)$ foi maior entre os HIV-positivos. Por outro lado, a taxa de isolamento de M. kansasii (17\%) foi maior entre os HIV-negativos, como observado em outros estudos realizados no Brasil, nos EUA e na Europa ${ }^{(14,16,20)}$.

A maioria (48\%) das culturas analisadas foi obtida a partir de espécimes de origem pulmonar, seguida pelo sangue e ou pela medula óssea (29\%). Considerando as duas espécies mais freqüentes, verificamos que o $M$. kansasii foi mais prevalente em sítio pulmonar (83\%) e o MAC, em doença disseminada (44,2\%). Estes dados coincidem com os da literatura mundial, que demonstram que $30 \%$ a $50 \%$ dos pacientes HIV-positivos apresentam doença disseminada causada por $\operatorname{MAC}^{(4,9,25)}$.

O isolamento em uma única amostra de sítios não-estéreis, como escarro, urina ou fezes, geralmente não tem significado clínico, sugerindo contaminação ou colonização transitória ${ }^{(11,23,28)}$. Neste estudo, considerando-se os isolamentos de sítios não-estéreis, observou-se que $79,8 \%$ dos pacientes tiveram apenas uma cultura positiva em amostra de origem pulmonar. Essa taxa foi ainda maior em amostras de urina $(95,3 \%)$ e fezes (100\%). Salientamos que a presença de MNT em uma única amostra de origem não-estéril requer investigação cuidadosa, e novas amostras devem ser examinadas para descartar a possibilidade de contaminação ou colonização transitória. Este estudo retrospectivo baseouse em resultados de culturas recebidas para identificação, e não foi possível resgatar os resultados da baciloscopia ou mesmo verificar se os pacientes tiveram outras culturas com resultados negativos. Setenta e quatro $(12,4 \%)$ pacientes tiveram duas culturas positivas de espécimes de origem pul- 


\section{Distribuição dos sítios de origem segundo o número de cepas isoladas por paciente}

Tabela 4 de 1991 a 1997

\begin{tabular}{|c|c|c|c|c|}
\hline \multirow{2}{*}{ Sítios de origem } & \multirow{2}{*}{$\begin{array}{c}n \text { total } \\
(n=1.248)\end{array}$} & \multicolumn{3}{|c|}{$n(\%)$ de cepas por paciente } \\
\hline & & 1 & 2 & 3 ou mais \\
\hline Pulmonar & 600 & $479(79,8)$ & $74(12,4)$ & $47(7,8)$ \\
\hline Disseminado & 366 & $194(53)$ & $92(25)$ & $80(22)$ \\
\hline Sangue/medula & 360 & $194(53,9)$ & $88(24,4)$ & $78(21,7)$ \\
\hline Dois ou mais sítios estéreis & 6 & - & $4(66,7)$ & $2(33,3)$ \\
\hline Extrapulmonar & 197 & $186(94,5)$ & $10(5)$ & $1(0,5)$ \\
\hline \multicolumn{5}{|l|}{ Sitios estéreis } \\
\hline Biópsia de gânglio & 29 & $27(93,1)$ & $2(6,9)$ & - \\
\hline Biópsia de fígado & 14 & $14(100)$ & - & - \\
\hline Líquido cefalorraquidiano & 11 & $11(100)$ & - & - \\
\hline Líquido pleural & 11 & $11(100)$ & - & - \\
\hline Biópsia de estômago & 4 & $4(100)$ & - & - \\
\hline \multicolumn{5}{|l|}{ Sitios não-estéreis } \\
\hline Urina & 85 & $81(95,3)$ & $4(4,7)$ & - \\
\hline Biópsia/secreção de pele & 22 & $19(86,4)$ & $3(13,6)$ & - \\
\hline Fezes & 17 & $17(100)$ & - & - \\
\hline Outros & 3 & $2(66,7)$ & $1(33,3)$ & - \\
\hline Dois ou mais sítios não-estéreis & 3 & - & $2(66,7)$ & $1(33,3)$ \\
\hline Um sítio estéril e outro não-estéril & 1 & - & $1(100)$ & - \\
\hline Ignorado & 81 & $73(90)$ & $4(5)$ & $4(5)$ \\
\hline
\end{tabular}

Pulmonar: escarro, lavado gástrico, lavado brônquico.

monar. Segundo a American Thoracic Society, duas culturas positivas e uma baciloscopia positiva confirmariam o diagnóstico bacteriológico. Somente 7,8\% dos casos tiveram o diagnóstico bacteriológico confirmado pelo isolamento da mesma espécie em três ou mais amostras.

Isolamentos de MNT potencialmente patogênicas em apenas uma amostra de sítios estéreis ou biópsias são indicativos de doença. Neste estudo, 436 (35\%) casos tiveram MNT isoladas de sítios estéreis e/ou biópsias, portanto o diagnóstico foi confirmado bacteriologicamente.

É importante ressaltar que os critérios para o diagnóstico das infecções causadas pelas MNT foram publicados por autores e instituições internacionais, e não há, no Brasil, recomendações oficiais sobre o assunto, o que auxiliaria esse diagnóstico.

Nas últimas décadas, metodologias de investigação de DNA permitiram o reconhecimento de muitas espécies novas. Desde 1990, 42 novas espécies de micobactérias foram descritas, a maioria isolada de espécimes $\operatorname{clínicos}^{(26)}$. As tabelas utilizadas na identificação fenotípica convencional foram elaboradas a partir dos estudos taxonômicos realizados nas décadas de 1970 e 1980 . Muitas espécies novas foram descritas com base em análise genética, e, por não terem sido caracterizadas fenotipicamente, não foram incluídas nas tabelas utilizadas na identificação fenotípica. Neste estudo foi utilizado somente o método fenotípico convencional, e dez espécies foram identificadas. Em conseqüência, 3,8\% dos pacientes não puderam ter diagnósticos elucidados, visto que as MNT isoladas não foram identificadas pela metodologia empregada. Embora seja um percentual baixo, não exclui sua importância. Muitas das novas espécies descritas são potencialmente patogênicas, e há numerosos relatos de sua associação com doença. Por essa razão, a implantação de métodos moleculares de 
identificação poderia esclarecer e permitir o diagnóstico correto destes casos.

\section{Conclusões}

No estado de São Paulo, na década de 1990, houve um aumento nas taxas de isolamento das MNT quando em comparação com décadas passadas. No entanto, é difícil a correlação entre os isolamentos e o diagnóstico da doença devido à inexistência de uma regulamentação oficial brasileira estabelecendo os critérios clínicos e bacteriológicos e, também, pela falta de integração entre a clínica e o laboratório, de modo a garantir os diversos isolamentos do mesmo sítio. As espécies de MNT mais freqüentemente isoladas foram MAC e M. kansasii. A aplicação de metodologias moleculares possibilitaria definir a diversidade das espécies de micobactérias que não puderam ser identificadas pelos métodos clássicos. Uma publicação nacional com recomendações sobre essas infecções é fundamental para a conduta correta no diagnóstico e no tratamento de micobacterioses.

\section{Referências}

I.ADLE-BIASSETTE, H. et al. Les mycobactéries non tuberculeuses. Ann Pathol, v 23, n. 3, p. 216-35, 2003.

2. AlLY, D. C. G. et al. Systemic mycobacterioses in AIDS patients as determined by blood cultures on biphasic medium. Rev Arg Microbiol, v. 31, p. 53-7, 1999.

3. AMERICANTHORACIC SOCIETY. Diagnosis and treatment of disease caused by nontuberculous mycobacteria. Am Rev Respir Dis, v. 142, p. 940-53, 1990.

4. AMERICANTHORACIC SOCIETY. Diagnosis and treatment of disease caused by nontuberculous mycobacteria. Am J Respir Crit Care Med, v. I56, p. sI-s25, 1997

5.BARRETO, A. M. W.; CAMPOS, C. E. D. Micobactérias nãotuberculosas no Brasil. Bol Pneum Sanit, v. 8, n. I, p. 23-32, 2000.

6. BARRETO, J. A. et al. Isolation of Mycobacterium avium complex from bone marrow aspirates of AIDS patients in Brazil. J Infect Dis, v. 168, p. 777-9, 1993.

7. CAMPOS, H. S. Manejo da doença micobacteriana não-tuberculosa. Bol Pneum Sanit, v. 8, n. 2, p. 39-50, 2000.

8. CENTRO PANAMERICANO DE ZOONOSIS. Manual de Normas y procedimientos técnicos para la bacteriología de la tuberculosis. Parte III. Sensibilidad del Mycobacterium tuberculosis a las drogas. La identificación de micobacteria. 1988.

9. CHIN, D. P. et al. Mycobacterium avium complex in the respiratory or gastrointestinal tract and the risk of M.avium complex bacteremia in patients with human immunodeficiency virus infection. I Infect Dis, v. 169, n. 2, p. 289-95, 1994.

I0. COLLINS, C. H.; GRANGE, J. M.;YATES, M. D. Identification of species. In:Tuberculosis bacteriology: organization and practice. 2. ed. Oxford: Butterworth-Heinemann, 1997. I39p.

I I. DAVIDSON, P.T.The diagnosis and management of disease caused by M. avium complex, M. kansasii and other mycobacteria. Clin Chest Med, v. 10, n. 3, p. 59-63, 1989.

12. DIAS, J. L. M. et al.Anti-retroviral therapy and public health: a balance of the brazilian experience. Disponível em: <http://www. sidalac.org.mx/english/publications/arv/brasil.pdf>. Acesso em 13/4/2004.
13. EUZÉBY, J. P. List of bacterial names with standing in nomenclature. Disponível em: <http://www.bacterio.cict. $\mathrm{fr} / \mathrm{m} /$ mycobacterium.htm $>$. Acesso em 9/3/2004.

| 4. FALKINHAM, J. O. Epidemiology of infection by nontuberculous mycobacteria. Clin Microbiol Rev, v. 9, n. 2, p. 177-215, 1996.

I5. FANDINHO, F. C. O. et al. Diagnosis of disseminated mycobacterial infection: proposal of a simple and nonexpensive method to be used in developing countries. Bull World Health Organ, v. 75, n. 4, p. 36I-6, 1997.

16. FERRAZOLI, L. et al. Micobactérias outras que não o Mycobacterium tuberculosis: análise da ocorrência e de aspectos relevantes ao diagnóstico da infecção. Hansen Int, v. 17, n. I/2, p. 15-20, 1992.

17. FERREIRA, R. M. C. et al. Non-tuberculous mycobacteria I: one year clinical isolates identification in Tertiary Hospital Aids Reference Center, Rio de Janeiro, Brazil, in pre highly active antiretroviral therapy era. Mem Inst Oswaldo Cruz, v. 97, n. 5, p. 725-9, 2002

18. HADAD, D. J. et al. Mycobacterium avium complex (MAC) isolated from aids patients and the criteria required for its implication in disease. Rev Inst Med Trop São Paulo, v. 37, n. 50, p. 375-83, 1995.

19. HORSBURGH JR., C. R.; SELIK, R. M. The epidemiology of disseminated nontuberculous mycobacterial infection in the acquired immunodeficiency syndrome (AIDS). Am Rev Respir Dis, v. 139, p. 4-7, 1989

20. MARTíNEZ-MORAGÓN, E. et al. Enfermedades por micobacterias ambientales en pacientes con y sin infección por el $\mathrm{VIH}$ : características epidemiológicas, clínicas y curso evolutivo. Arch Bronconeumol, v. 37, p. 28 I-6, 200 I.

21. MINISTÉRIO DA SAÚDE. Fundação Nacional de Saúde. Centro de Referência Professor Hélio Fraga. Manual de Bacteriologia da Tuberculose. 2. ed., Rio de Janeiro, 1994, I I 5p.

22. OPRAVIL, M. Epidemiological and clinical aspects of mycobacterial infections. Infection, v. 25, n. I, p. 56-9, 1997.

23. RAJU, B.; SCHLUGER, N.W. Significance of respiratory isolates of Mycobacterium avium complex in HIV-positive and HIVnegative patients. Int J Infect Dis, 4: I34-9, 2000.

24. SECRETARIA DE ESTADO DA SAÚDE DE SÃO PAULO. Centro de Referência e Treinamento DST/AIDS, Centro 
UEKI, S. Y. M. et al. Micobactérias não-tuberculosas: diversidade das espécies no estado de São Paulo •| Bras Patol Med Lab •v. $41 \bullet$ n. 1 •p. 1-8 • fevereiro 2005

de Vigilância Epidemiológica. Boletim Epidemiológico, v. XVII, n. 2, 1999.

25. THE RESEARCH COMMITTEE OF THE BRITISH THORACIC SOCIETY. Pulmonary disease caused by Mycobacterium avium-intracellulare in HIV-negative patients: five-year follow-up of patients receiving standardised treatment. Int J Tuberc Lung Dis, v. 6, n. 7, p. 628-34, 2002.
26. TORTOLI, E. Impact of genotypic studies on mycobacterial taxonomy: the new mycobacteria of the 1990s. Clin Microbiol Rev, v. 16, p. 319-54, 2003.

27.TSUKAMURA, M. Identification of mycobacteria. Obu, Aichi:The National Chubu Hospital, Japan, 1984.

28. VAN CREVEL, R. et al. The impact of nontuberculous mycobacteria on management of presumed pulmonary tuberculosis. Infection, v. 29, n. 2, p. 59-63, 200I. 